\title{
Clinical Significance of Expression of Immunoadjuvant Molecules (LAG-3, TIM-3, OX-40) in Neoadjuvant Chemotherapy for Breast Cancer
}

\author{
Yuka Asano \\ Osaka City University Graduate School of Medicine \\ Shinichiro Kashiwagi ( $\sim$ spqv9ke9@view.ocn.ne.jp) \\ Osaka City University Graduate School of Medicine \\ Sae Ishihara \\ Osaka City University Graduate School of Medicine \\ Koji Takada \\ Osaka City University Graduate School of Medicine \\ Wataru Goto \\ Osaka City University Graduate School of Medicine \\ Tamami Morisaki \\ Osaka City University Graduate School of Medicine \\ Masatsune Shibutani \\ Osaka City University Graduate School of Medicine \\ Hiroaki Tanaka \\ Osaka City University Graduate School of Medicine \\ Kosei Hirakawa \\ Osaka City University Graduate School of Medicine \\ Masaichi Ohira \\ Osaka City University Graduate School of Medicine
}

\section{Research Article}

Keywords: immunoadjuvant molecules, breast cancer, pathological complete response, neoadjuvant chemotherapy, LAG-3, TIM-3, OX40

Posted Date: August 13th, 2021

DOl: https://doi.org/10.21203/rs.3.rs-785791/v1

License: @ (1) This work is licensed under a Creative Commons Attribution 4.0 International License. Read Full License

Version of Record: A version of this preprint was published at Anticancer Research on December 29th, 2021. See the published version at https://doi.org/10.21873/anticanres.15466. 


\section{Abstract \\ Purpose}

Various immunosuppressive factors that inhibit the immune response to cancer are present in cancer cells and the cancer microenvironment. Co-inhibitory and co-stimulatory receptors are dynamically expressed on T-cells as immunoadjuvant molecules that regulate the state of T-cell activity. In this report we focus on immunoadjuvant molecules such as LAG-3, TIM-3, and OX-40, for which there have been few published reports. We investigated the expression of LAG-3, TIM-3, and OX-40 in tumor-infiltrating lymphocytes (TILs), and clinically verified the significance of that expression in relation to neoadjuvant thermotherapy (NAC).

\section{Methods}

A total of 177 patients with resectable early-stage breast cancer were treated with NAC. Estrogen receptor (ER), progesterone receptor (PgR), human epidermal growth factor receptor 2 (HER2), Ki67, LAG-3, TIM-3 and OX-40 status were assessed by immunohistochemistry.

\section{Results}

There were 47 (26.6\%) patients with high LAG-3 expression, 31 (17.5\%) patients had high TIM-3 expression, and 32 (18.1\%) patients had high OX-40 expression. The group with low-LAG-3 expression was significantly smaller than the group with high expression in triple-negative breast cancer (TNBC) $(p=0.038)$ and HER2-enriched breast cancer (HER2BC) $(p=0.021)$, and the total number of pathological complete response ( $\mathrm{PCR}$ ) patients was greater $(p<0.001)$. In TNBC and HER2BC, the pCR rate was significantly higher in the low-LAG-3 expression group than in the high-LAG-3 expression group $(p<0.001)(p=0.020)$. Moreover, on multivariate analysis also showed that low-LAG-3 expression status was an independent factor to predict the favorable prognosis $(p=0.014, H R=8.124)(p$ $=0.048, \mathrm{HR}=10.400)$.

\section{Conclusions}

Our findings suggest that LAG-3 may become a biomarker in highly malignant breast cancers such as TNBC and HER2BC that can predict the therapeutic efficacy of NAC.

\section{Background}

Various immunosuppressive factors that inhibit the immune response to cancer are present in cancer cells and the cancer microenvironment $[1,2]$. Co-inhibitory (suppression) and co-stimulatory receptors (activation) are dynamically expressed on T-cells as immunoadjuvant molecules that regulate the state of T-cell activity, and the inhibitory molecules act as immunological checkpoints. There are a number of immunological checkpoints in the progression of the immune response, and the co-inhibitory functions of receptors such as cytotoxic T-lymphocyte-associated antigen 4 (CTLA-4) and programmed cell death 1 (PD-1: CD279) are considered particularly important checkpoints for regulating the autoimmune response [3,4]. Antitumor effects of immunological checkpoint inhibition therapy utilizing an anti-CTLA-4 antibody and an anti-PD-1 antibody as antitumor T-cell effectors have been discovered in many types of cancers, and these findings have dramatically changed the positioning of cancer immunotherapy in clinical settings [5-8]. In breast cancer (BC) as well, it has been reported that the anti-PD-1 antibody pembrolizumab has antitumor activity toward triple-negative breast cancer (TNBC), and that the levels of expression of PD-1 and its ligand, programmed cell death-ligand-1 (PDL-1: CD274), correlate with prognosis [9-12]. As a result, these findings can be expected to affect individualized therapy for breast cancer in the future. We have previously suggested that the expression of PD-1 and PD-L1 may serve as biomarkers for predicting therapeutic efficacy in neoadjuvant chemotherapy (NAC) for breast cancer. In this report we focus on immunoadjuvant molecules such as lymphocyte activation gene-3 (LAG-3: CD223), T-cell immunoglobulin and mucin containing protein-3 (TIM-3), and orexin-40 (OX-40: CD134), for which there have been few published reports. LAG-3 works as a molecule that suppresses the proliferation and activation of T-cells, and it is also expressed by tumor-infiltrating lymphocytes (TILs), T-cells that have been impoverished by chronic infection (exhausted T cells), and by regulatory T cells (Tregs) [13-15]. TIM-3 not only functions as an immunological checkpoint regulator that suppresses T-cell function synergistically with PD-1, but it also suppresses the natural immune response of myeloid-lineage cells and 
is responsible for important functions in cancer cell support and survival [16-18]. OX-40 is an immuno-co-stimulatory molecule that is expressed by activated T-cells, is expressed by Tregs, and supports memory T-cells [19-21].

The importance of regulating and improving the immune microenvironment in cancer has been recognized because the immune microenvironment in cancer tissues affects not only the efficacy of immunotherapy, but also the efficacy and prognosis of conventional chemotherapy and other modes of anticancer therapy $[22,23]$. Therefore, monitoring the host's immune response to cancer in the microenvironment is believed to play a key role in predicting therapeutic efficacy and prognosis. TILs, which are considered indicators of immune response monitoring, have been reported as prognostic factors and predictors of therapeutic efficacy [24-26]. In this study, we investigated the expression of LAG-3, TIM-3, and OX-40 in TILs, and clinically verified the significance of that expression in relation to NAC.

\section{Methods}

\section{Patient Background}

A total of 177 patients with resectable early-stage breast cancer diagnosed as stage IIA (T1, N1, M0 or T2, N0, M0), IIB (T2, N1, M0 or $\mathrm{T} 3, \mathrm{~N} 0, \mathrm{M} 0)$ or IIIA (T1-2, N2, M0 or T3, N1-2, M0) were treated with NAC between 2007 and 2013. Tumor stage and T and N factors were stratified based on the TNM Classification of Malignant Tumours, UICC Seventh Edition [27]. Breast cancer was confirmed histologically by core needle biopsy and staged by systemic imaging studies using computed tomography (CT), ultrasonography (US) and bone scintigraphy. Breast cancer was classified into subtypes according to the immunohistochemical expression of estrogen receptor (ER), progesterone receptor (PgR), human epidermal growth factor receptor 2 (HER2) and Ki67. Based on their immunohistochemical expression, the tumors are categorized into the immuno-phenotypes of luminal A (ER + and/or PgR+, HER2-, Ki67-low), luminal B (ER + and/or PgR+, HER2+) (ER + and/or PgR+, HER2-, Ki67-high), HER2-enriched breast cancer (HER2BC) (ER-, PgR-, and HER2+), and TNBC (negative for ER, PgR and HER2). In this study, luminal A and luminal $B$ were assumed to be hormone receptor-positive breast cancer (HRBC).

All patients received a standardized protocol of NAC consisting of four courses of FEC100 $\left(500 \mathrm{mg} / \mathrm{m}^{2}\right.$ fluorouracil, $100 \mathrm{mg} / \mathrm{m}^{2}$ epirubicin and $500 \mathrm{mg} / \mathrm{m}^{2}$ cyclophosphamide) every 3 weeks, followed by 12 courses of paclitaxel $\left(80 \mathrm{mg} / \mathrm{m}^{2}\right)$, administered weekly $[28,29]$. Forty-five patients were diagnosed with HER2-positive breast cancer and trastuzumab was administered on a weekly (2 $\mathrm{mg} / \mathrm{kg}$ ) or tri-weekly $(6 \mathrm{mg} / \mathrm{kg}$ ) basis, during paclitaxel treatment [30]. All patients underwent chemotherapy as outpatients. Therapeutic anti-tumour effects were assessed according to the Response Evaluation Criteria in Solid Tumors (RECIST) criteria [31]. Pathological complete response ( $\mathrm{pCR}$ ) was defined as the complete disappearance of the invasive compartment of the lesion with or without intraductal components, including the lymph nodes. Patients underwent mastectomy or breast-conserving surgery after NAC. All patients who underwent breast-conserving surgery were administered postoperative radiotherapy to the remnant breast. We operated for seven cases of progressive disease (PD) during the NAC enforcement when progression was confirmed. Overall survival (OS) was defined as the period from the initiation of NAC to the time of death from any cause. Disease-free interval (DFS) was defined as the interval in years from the date of the primary surgery to the first local recurrence, distant recurrences, or death from any cause. All patients were followed up by physical examination every 3 months, US every 6 months and CT and bone scintigraphy annually. The median follow-up period for the assessment of OS was 3.4 years (range, 0.6-6.0 years) and 3.1 years for DFS (range, 0.1-6.0 years). The design of this study is a retrospective chart review study. Written informed consent was obtained from all subjects. This research conformed to the provisions of the Declaration of Helsinki in 1995. All patients were informed of the investigational nature of this study and provided their written, informed consent. The study protocol was approved by the Ethics Committee of Osaka City University (\#926).

\section{Immunohistochemistry}

All patients underwent a core needle biopsy prior to NAC, and they had undergone a curative operation involving a mastectomy or conservative surgery with axillary lymph node dissection after NAC at Osaka City University. Immunohistochemical studies were performed as previously described on core needle biopsy specimens [32]. Tumor specimens were fixed in $10 \%$ formaldehyde solution, embedded in paraffin, and 4- $\mu \mathrm{m}$-thick sections were mounted onto glass slides. Slides were deparaffinized in xylene and heated for 20 $\min \left(105^{\circ} \mathrm{C}, 0.4 \mathrm{~kg} / \mathrm{m}^{2}\right)$ in an autoclave in Target Retrieval Solution (Dako, Carpinteria, CA, USA). Specimens were then incubated with $3 \%$ hydrogen peroxide in methanol for 15 min to block endogenous peroxidase activity, followed by incubation in $10 \%$ normal goat or rabbit serum to block non-specific reactions. 
Primary monoclonal antibodies directed against ER (clone 1D5, dilution 1:80; Dako, Cambridge, UK), PgR (clone PgR636, dilution 1:100; Dako), HER2 (HercepTest ${ }^{\text {TM}}$; Dako), Ki67 (clone MIB-1, dilution 1:00; Dako), LAG-3 (clone 11E3, dilution 1:100, Abcam), TIM-3 (polyclonal, dilution 1:100, Abcam, Cambridge, UK), and OX-40 (clone ACT35, dilution 1:100, BD Biosciences, Franklin Lakes, NJ, USA) were used. Tissue sections were incubated with each antibody for $70 \mathrm{~min}$ at room temperature or overnight at $4^{\circ} \mathrm{C}$, and were then incubated with horseradish peroxidase-conjugated anti-rabbit or anti-mouse lg secondary antibodies (HISTOFINE (PO) ${ }^{\mathrm{Tm}}$ kit; Nichirei, Tokyo, Japan). Slides were subsequently treated with streptavidin-peroxidase reagent and were then incubated in phosphate-buffered saline-diaminobenzidine and $1 \%$ hydrogen peroxide $(\mathrm{v} / \mathrm{v})$, followed by counterstaining with Mayer's hematoxylin. Positive and negative controls for each marker were used according to the supplier's data sheet.

\section{Immunohistochemical scoring}

Immunohistochemical scoring was performed by two pathologists specialized in mammary gland pathology, using the blind method to confirm the objectivity and reproducibility of diagnosis. The cut-off value for ER and PgR positivity was set at $\geq 1 \%$ in accordance with previous studies [33]. HER2 expression was scored according to the accepted grading system (0, no reactivity or membranous reactivity in less than $10 \%$ of cells; $1+$, faint/barely perceptible membranous reactivity in $\geq 10 \%$ of cells or reactivity in only part of the cell membrane; $2+$, weak to moderate complete or basolateral membranous reactivity in $\geq 10 \%$ of tumor cells; or $3+$, strong complete or basolateral membranous reactivity in $\geq 10 \%$ of tumor cells). HER2 expression was considered positive if the immunostaining score was $3+$, or in cases where the score was $2+$ and included gene amplification determined using fluorescent in situ hybridization (FISH). For FISH analyses, each copy of the HER2 gene and its centromere 17 (CEP17) reference were counted. The interpretation followed the criteria of the ASCO/CAP guidelines for HER2 IHC classification for breast cancer: considered positive if the HER2/CEP17 ratio was higher than $2.0[34,35]$. A Ki67-labeling index with $\geq 14 \%$ of tumor cells with nuclear staining was considered positive [36].

Histopathological analysis of the percentage of TILs was evaluated on a single full-face hematoxylin and eosin (HE)-stained tumor section using criteria described by Salgado et al [37]. TILs were defined as the infiltrating lymphocytes within tumor stroma and are expressed by the proportion of the field investigated [38, 39]. To evaluate LAG-3, TIM-3, and OX-40 expression, the number of infiltrating T-lymphocytes stained with anti-LAG-3, anti-TIM-3, and anti-OX-40 antibody in areas surrounding cancer cells was measured under 400-times magnified microscopy in each of 5 fields of view (FOVs) selected in darkly stained areas (Fig. 1A, B, C). Based on previous reports as a reference, the median value of the average number in the 5 FOVs was determined, and that number was set as a cut-off value $[9,15,37]$.

\section{Statistical analysis}

Statistical analysis was performed using the SPSS ${ }^{\circledR}$ version 19.0 statistical software package (IBM, Armonk, NY, USA). Categorical data are reported with numbers and percentages, and continuous data as a median and range. The association between LAG-3, TIM-3, OX-40, and clinicopathological variables, and the significance of different prognostic markers were analyzed using the chi-square test (or Fisher's exact test when necessary). Association with survival was analyzed using the Kaplan-Meier plot and the log-rank test. The Cox proportional hazards model was used to compute univariable and multivariable hazards ratios (HR) for the study parameters with a 95\% confidence interval (c.i.), and was used in a backward stepwise method for variable selection in multivariate analyses. In all of the tests, a $p$-value of less than 0.05 was considered statistically significant. Cut-off values for different biomarkers included in this study were chosen before statistical analysis.

\section{Results}

\section{Clinicopathological responses of primary breast cancers to NAC}

The BC subtypes among the 177 patients who received NAC were: TNBC in 61 (34.5\%), HER2BC in 36 (20.3\%), and HRBC in 80 (45.2\%) patients. Treatment response was: PCR in 67 (37.9\%), partial response (PR) in 84 (47.5\%), stable disease (SD) in 19 (10.7\%) and PD in 7 (3.9\%) patients. Based on subtype, pCR was achieved in 28 (45.9\%) TNBC, 18 (50.0\%) HER2BC, and 21 (26.3\%) HRBC patients.

\section{Expression of immunoadjuvant molecules in all breast cancer patients}

Among the total number of breast cancer patients $(n=177), 47$ patients $(26.6 \%$; range: 0 to $46 \%$; mean, $8 \%$; median, $7 \%$; standard deviation $5 \%$ ) tested positive for LAG-3 expression, 31 patients (17.5\%; range: 0 to $37 \%$; mean, $6 \%$; median, $6 \%$; standard deviation $4 \%$ ) tested positive for TIM-3 expression, and 32 patients (18.1\%; range: 0 to 38\%; mean, 7\%; median, $8 \%$; standard deviation $4 \%$ ) tested

Page 4/17 
positive for OX-40 expression. When the clinicopathological features were examined, the group with low-LAG-3 expression was significantly smaller than the group with high expression in TNBC $(p=0.038)$ and HER2BC $(p=0.021)$, but was significantly larger in HRBC $(p<0.001)$, and the total number of $p C R$ patients was greater $(p<0.001)$. In the TIM-3 low-expression group, tumor diameter was significantly smaller $(p=0.007)$ and the Ki-67 level was significantly higher $(p=0.043)$ than in the high-expression group, and no correlation was found between intrinsic subtype and the PCR rate. OX-40 expression also showed no correlation between intrinsic subtype and the pCR rate (Table 1). In the prognostic analysis, we found a significant DFS extension in the LAG-3 low-expression group over the high-expression group ( $p=0.001$, log-rank) (Fig. 2A). We also found a significant OS extension in the LAG-3 lowexpression group over the high expression group $(p<0.001)(F i g .3 A)$. There was no significant difference in DFS and OS between TIM3 and 0X-40 expression level groups (Supplemental Fig. 1A-D). In the univariate analysis, low-LAG-3 expression significantly contributed to extension of the disease-free survival interval $(p=0.002, H R=3.239)$. Low-LAG-3 expression was also an independent factor for good prognosis $(p=0.006, H R=3.047)$ in the multivariate analysis (Table 2 ). 
Table 1

Correlation between clinicopathological features and LAG-3, TIM-3, and OX-40 expression in 177 all breast cancers.

\begin{tabular}{|c|c|c|c|c|c|c|c|c|c|}
\hline \multirow[t]{2}{*}{ Parameters } & \multicolumn{2}{|l|}{ LAG-3 } & \multirow{2}{*}{$\begin{array}{l}p \\
\text { value }\end{array}$} & \multicolumn{2}{|l|}{ TIM-3 } & \multirow{2}{*}{${ }_{\text {value }}^{p}$} & \multicolumn{2}{|l|}{$0 X-40$} & \multirow{2}{*}{$\begin{array}{l}p \\
\text { value }\end{array}$} \\
\hline & $\begin{array}{l}\text { Positive } \\
(n=47)\end{array}$ & $\begin{array}{l}\text { Negative } \\
(n=130)\end{array}$ & & $\begin{array}{l}\text { Positive } \\
(n=31)\end{array}$ & $\begin{array}{l}\text { Negative } \\
(n=146)\end{array}$ & & $\begin{array}{l}\text { Positive } \\
(n=32)\end{array}$ & $\begin{array}{l}\text { Negative } \\
(n=145)\end{array}$ & \\
\hline \multirow{2}{*}{$\begin{array}{l}\text { Age at } \\
\text { operation } \\
\leq 56\end{array}$} & $18(38.3 \%)$ & $69(53.1 \%)$ & \multirow[t]{3}{*}{0.082} & $14(45.2 \%)$ & $73(50.0 \%)$ & \multirow[t]{3}{*}{0.625} & $16(50.0 \%)$ & 71 (49.0\%) & \multirow[t]{3}{*}{0.916} \\
\hline & $29(61.7 \%)$ & $61(46.9 \%)$ & & $17(54.8 \%)$ & $73(50.0 \%)$ & & $16(50.0 \%)$ & 74 (51.0\%) & \\
\hline \multicolumn{7}{|l|}{$>56$} & & & \\
\hline Menopause & 15 (31.9\%) & 57 (43.8\%) & \multirow[t]{3}{*}{0.154} & $12(38.7 \%)$ & $60(41.1 \%)$ & \multirow[t]{3}{*}{0.806} & 12 (37.5\%) & $60(41.4 \%)$ & \multirow[t]{3}{*}{0.686} \\
\hline Negative & $32(68.1 \%)$ & 73 (56.2\%) & & 19 (61.3\%) & $86(58.9 \%)$ & & $20(62.5 \%)$ & 85 (58.6\%) & \\
\hline \multicolumn{7}{|l|}{ Positive } & & & \\
\hline Tumor size & $5(10.6 \%)$ & 19 (14.6\%) & \multirow[t]{2}{*}{0.495} & $0(0.0 \%)$ & $24(16.4 \%)$ & \multirow[t]{2}{*}{0.007} & $4(12.5 \%)$ & 20 (13.8\%) & \multirow[t]{2}{*}{0.554} \\
\hline $\begin{array}{l}\leq 2 \mathrm{~cm} \\
>2 \mathrm{~cm}\end{array}$ & $42(89.4 \%)$ & $\begin{array}{l}111 \\
(85.4 \%)\end{array}$ & & $\begin{array}{l}31 \\
(100.0 \%)\end{array}$ & $\begin{array}{l}122 \\
(83.6 \%)\end{array}$ & & $28(87.5 \%)$ & $\begin{array}{l}125 \\
(86.2 \%)\end{array}$ & \\
\hline \multirow{2}{*}{$\begin{array}{l}\text { Lymph node } \\
\text { status } \\
\text { Negative }\end{array}$} & $12(25.5 \%)$ & 29 (22.3\%) & \multirow[t]{3}{*}{0.653} & $10(32.3 \%)$ & $31(21.2 \%)$ & \multirow[t]{3}{*}{0.186} & $9(28.1 \%)$ & 32 (22.1\%) & \multirow[t]{3}{*}{0.462} \\
\hline & 35 (74.5\%) & $\begin{array}{l}101 \\
(77.7 \%)\end{array}$ & & $21(67.7 \%)$ & $\begin{array}{l}115 \\
(78.8 \%)\end{array}$ & & $23(71.9 \%)$ & $\begin{array}{l}113 \\
(77.9 \%)\end{array}$ & \\
\hline \multicolumn{7}{|l|}{ Positive } & & & \\
\hline Nuclear grade & $38(80.9 \%)$ & 99 (76.2\%) & \multirow[t]{2}{*}{0.509} & $24(77.4 \%)$ & \multirow{2}{*}{$\begin{array}{l}113 \\
(77.4 \%) \\
33(22.6 \%)\end{array}$} & \multirow[t]{2}{*}{0.998} & $25(78.1 \%)$ & \multirow{2}{*}{$\begin{array}{l}112 \\
(77.2 \%) \\
33(22.8 \%)\end{array}$} & \multirow[t]{2}{*}{0.914} \\
\hline $\begin{array}{l}1,2 \\
3\end{array}$ & 9 (19.1\%) & $31(23.8 \%)$ & & $7(22.6 \%)$ & & & 7 (21.9\%) & & \\
\hline Ki67 & $23(48.9 \%)$ & $51(39.2 \%)$ & \multirow[t]{3}{*}{0.248} & $18(58.1 \%)$ & $56(38.4 \%)$ & \multirow[t]{3}{*}{0.043} & $11(34.4 \%)$ & $63(43.3 \%)$ & \multirow[t]{3}{*}{0.346} \\
\hline$\leq 14 \%$ & $24(51.1 \%)$ & 79 (60.8\%) & & $13(41.9 \%)$ & 90 (61.6\%) & & $21(65.6 \%)$ & $82(56.7 \%)$ & \\
\hline$>14 \%$ & & & & & & & & & \\
\hline HR and HER2 & $22(46.8 \%)$ & 39 (30.0\%) & 0.038 & $9(29.0 \%)$ & 52 (35.6\%) & 0.484 & $11(34.3 \%)$ & 50 (34.5\%) & 0.991 \\
\hline $\begin{array}{l}\text { TNBC } \\
\text { non-TNBC }\end{array}$ & $25(53.2 \%)$ & 91 (70.0\%) & & 22 (71.0\%) & $94(64.4 \%)$ & & $21(65.6 \%)$ & 95 (65.5\%) & \\
\hline Intrinsic & 15 (31.9\%) & $21(16.2 \%)$ & 0.021 & $5(16.1 \%)$ & $31(21.2 \%)$ & 0.521 & $6(18.7 \%)$ & $30(20.7 \%)$ & 0.805 \\
\hline $\begin{array}{l}\text { HER2BC } \\
\text { non-HER2BC }\end{array}$ & 32 (68.1\%) & $\begin{array}{l}109 \\
(83.8 \%)\end{array}$ & & $26(83.9 \%)$ & $\begin{array}{l}115 \\
(78.8 \%)\end{array}$ & & $26(81.3 \%)$ & $\begin{array}{l}115 \\
(79.3 \%)\end{array}$ & \\
\hline Intrinsic & $10(21.3 \%)$ & 70 (53.8\%) & $<$ & $17(54.8 \%)$ & $63(43.2 \%)$ & 0.235 & $15(46.9 \%)$ & $65(44.8 \%)$ & 0.833 \\
\hline $\begin{array}{l}\text { HRBC } \\
\text { non-HRBC }\end{array}$ & 37 (78.7\%) & $60(46.2 \%)$ & & $14(45.2 \%)$ & $83(56.8 \%)$ & & 17 (53.1\%) & 80 (55.2\%) & \\
\hline
\end{tabular}

TNBC, triple-negative breast cancer. HER2BC, human epidermal growth factor receptor 2-enriched breast cancer. HRBC, hormone receptor-positive breast cancer. pCR, pathological complete response. LAG-3, Lymphocyte activation gene-3. TIM-3, T-cell immunoglobulin and mucin containing protein-3. OX-40, orexin-40. 


\begin{tabular}{|c|c|c|c|c|c|c|c|c|c|}
\hline \multirow[t]{2}{*}{ Parameters } & \multicolumn{2}{|l|}{ LAG-3 } & \multirow{2}{*}{$\begin{array}{l}p \\
\text { value }\end{array}$} & \multicolumn{2}{|l|}{ TIM-3 } & \multirow{2}{*}{$\begin{array}{l}p \\
\text { value }\end{array}$} & \multicolumn{2}{|l|}{$0 X-40$} & \multirow{2}{*}{$p$} \\
\hline & $\begin{array}{l}\text { Positive } \\
(n=47)\end{array}$ & $\begin{array}{l}\text { Negative } \\
(n=130)\end{array}$ & & $\begin{array}{l}\text { Positive } \\
(n=31)\end{array}$ & $\begin{array}{l}\text { Negative } \\
(n=146)\end{array}$ & & $\begin{array}{l}\text { Positive } \\
(n=32)\end{array}$ & $\begin{array}{l}\text { Negative } \\
(n=145)\end{array}$ & \\
\hline \multirow{2}{*}{$\begin{array}{l}\text { Pathological } \\
\text { response } \\
\text { pCR }\end{array}$} & $7(14.9 \%)$ & $60(46.2 \%)$ & \multirow{2}{*}{$<.001$} & 10 (32.3\%) & 57 (39.0\%) & \multirow[t]{2}{*}{0.479} & $9(28.1 \%)$ & $58(40.0 \%)$ & \multirow[t]{3}{*}{0.210} \\
\hline & 40 (85.1\%) & 70 (53.8\%) & & 21 (67.7\%) & $89(61.0 \%)$ & & 23 (71.9\%) & $87(60.0 \%)$ & \\
\hline non-pCR & & & & & & & & & \\
\hline LAG-3 & \multirow{3}{*}{$\begin{array}{l}\text { Not } \\
\text { determined }\end{array}$} & \multirow{3}{*}{$\begin{array}{l}\text { Not } \\
\text { determined }\end{array}$} & & $24(77.4 \%)$ & \multirow{3}{*}{$\begin{array}{l}106 \\
(72.6 \%) \\
40(27.4 \%)\end{array}$} & \multirow[t]{3}{*}{0.581} & $23(71.9 \%)$ & \multirow{3}{*}{$\begin{array}{l}107 \\
(73.8 \%) \\
38(26.2 \%)\end{array}$} & \multirow[t]{3}{*}{0.824} \\
\hline Negative & & & & \multirow{2}{*}{$7(22.6 \%)$} & & & \multirow{2}{*}{$9(28.1 \%)$} & & \\
\hline Positive & & & & & & & & & \\
\hline TIM-3 & 40 (85.1\%) & \multirow{3}{*}{$\begin{array}{l}106 \\
(81.5 \%) \\
24(18.5 \%)\end{array}$} & \multirow[t]{3}{*}{0.581} & \multirow{3}{*}{$\begin{array}{l}\text { Not } \\
\text { determined }\end{array}$} & \multirow{3}{*}{$\begin{array}{l}\text { Not } \\
\text { determined }\end{array}$} & & 27 (84.4\%) & \multirow{3}{*}{$\begin{array}{l}119 \\
(82.1 \%) \\
26(17.9 \%)\end{array}$} & \multirow[t]{3}{*}{0.756} \\
\hline Negative & \multirow[t]{2}{*}{$7(14.9 \%)$} & & & & & & 5 (15.6\%) & & \\
\hline Positive & & & & & & & & & \\
\hline $0 X-40$ & 38 (80.9\%) & \multirow{2}{*}{$\begin{array}{l}107 \\
(82.3 \%)\end{array}$} & \multirow[t]{3}{*}{0.824} & $26(83.9 \%)$ & $\begin{array}{l}119 \\
(81.5 \%)\end{array}$ & \multirow[t]{3}{*}{0.756} & \multirow{3}{*}{$\begin{array}{l}\text { Not } \\
\text { determined }\end{array}$} & \multirow{3}{*}{$\begin{array}{l}\text { Not } \\
\text { determined }\end{array}$} & \\
\hline Negative & \multirow[t]{2}{*}{9 (19.1\%) } & & & \multirow[t]{2}{*}{$5(16.1 \%)$} & \multirow{2}{*}{27 (18.5\%) } & & & & \\
\hline Positive & & $20(11.110)$ & & & & & & & \\
\hline $\begin{array}{l}\text { TNBC, triple-r } \\
\text { receptor-posi } \\
\text { immunoglob }\end{array}$ & $\begin{array}{l}\text { e breast ce } \\
\text { d mucin cance }\end{array}$ & $\begin{array}{l}\text { r. HER2BC, } \\
\text { R, patholog } \\
\text { ining protei }\end{array}$ & $\begin{array}{l}\operatorname{man} \text { ep } \\
\text { l comp }\end{array}$ & $\begin{array}{l}\text { ermal growtl } \\
\text { te response. }\end{array}$ & $\begin{array}{l}\text { factor recept } \\
\text { AG-3, Lymph }\end{array}$ & $\begin{array}{l}\text { r 2-enri } \\
\text { cyte ac }\end{array}$ & $\begin{array}{l}\text { ed breast ca } \\
\text { ation gene- }\end{array}$ & $\begin{array}{l}\text { er. HRBC, horn } \\
\text { IM-3, T-cell }\end{array}$ & \\
\hline
\end{tabular}


Table 2

Univariable and multivariable analysis with respect to disease-free survival in breast cancer subtypes.

\begin{tabular}{|c|c|c|c|c|c|c|}
\hline \multirow[b]{2}{*}{ Parameter } & \multicolumn{3}{|c|}{ Univariable analysis } & \multicolumn{3}{|c|}{ Multivariable analysis } \\
\hline & $\begin{array}{l}\text { Hazard } \\
\text { ratio }\end{array}$ & 95 \% c.i. & $\begin{array}{l}\mathrm{p} \\
\text { value }\end{array}$ & $\begin{array}{l}\text { Hazard } \\
\text { ratio }\end{array}$ & 95 \% c.i. & $\mathrm{p}$ value \\
\hline & \multicolumn{6}{|c|}{ all breast cancers $(n=177)$} \\
\hline $\begin{array}{l}\text { Pathological response pCR vs non- } \\
\text { pCR }\end{array}$ & 0.611 & $0.279-1.336$ & 0.217 & 0.810 & $0.357-1.840$ & 0.615 \\
\hline LAG-3 & 3.239 & $1.522-6.892$ & 0.002 & 3.047 & $1.384-6.707$ & 0.006 \\
\hline \multicolumn{7}{|l|}{ Positive vs Negative } \\
\hline & \multicolumn{6}{|c|}{ TNBC $(\mathrm{n}=61)$} \\
\hline $\begin{array}{l}\text { Pathological response pCR vs non- } \\
\text { pCR }\end{array}$ & 0.234 & $0.050-1.084$ & 0.063 & 0.602 & $0.113-3.207$ & 0.552 \\
\hline LAG-3 & 10.107 & $2.170-47.083$ & 0.003 & 8.124 & $1.520-43.420$ & 0.014 \\
\hline \multicolumn{7}{|l|}{ Positive vs Negative } \\
\hline & \multicolumn{6}{|c|}{ HER2BC $(n=36)$} \\
\hline $\begin{array}{l}\text { Pathological response pCR vs non- } \\
\text { pCR }\end{array}$ & 0.464 & $0.077-2.802$ & 0.402 & 0.549 & $0.089-3.401$ & 0.519 \\
\hline LAG-3 & 10.969 & $\begin{array}{l}1.093- \\
110.131\end{array}$ & 0.042 & 10.400 & $\begin{array}{l}1.023- \\
105.734\end{array}$ & 0.048 \\
\hline & \multicolumn{6}{|c|}{$\operatorname{HRBC}(\mathrm{n}=80)$} \\
\hline $\begin{array}{l}\text { Pathological response pCR vs non- } \\
\text { pCR }\end{array}$ & 1.325 & $0.443-3.965$ & 0.614 & 1.261 & $0.412-3.860$ & 0.684 \\
\hline LAG-3 & 0.628 & $0.081-4.846$ & 0.655 & 0.681 & $0.085-5.493$ & 0.719 \\
\hline \multicolumn{7}{|l|}{ Positive vs Negative } \\
\hline
\end{tabular}

\section{LAG-3 expression in triple-negative breast cancer}

Among the 61 TNBC patients, 22 (36.1\%) were in the high-LAG-3 expression group, and 39 (63.9\%) were in the low-LAG-3 expression group. The $p C R$ rate was significantly higher $(p<0.001)$ in the low-LAG-3 expression group than in the high-LAG-3 expression group (Table 3). Outcome analysis showed significantly longer DFS ( $<<0.001$, log-rank) (Fig. $2 B)$ and OS $(p=0.003$, log-rank) (Fig. 3B) in the low-LAG-3 expression group than that in the high-LAG-3 expression group. Low-LAG-3 expression group in patients with TNBC demonstrated significantly lower rate for the recurrence by univariate $(p=0.003, H R=10.107)$ and multivariate analysis $(p=0.014, \mathrm{HR}$ $=8.124)$ (Table 2). 
Table 3

Correlations between LAG-3 expression and clinicopathological parameters in 61 triple-negative, 36 HER2 enriched, and 80 Luminaltype breast cancers.

\begin{tabular}{|c|c|c|c|c|c|c|c|c|c|}
\hline \multirow[t]{2}{*}{ Parameters } & \multicolumn{2}{|c|}{ TNBC $(n=61)$} & \multirow{2}{*}{$\begin{array}{l}p \\
\text { value }\end{array}$} & \multicolumn{2}{|c|}{ HER2BC $(n=36)$} & \multirow{2}{*}{$\begin{array}{l}p \\
\text { value }\end{array}$} & \multicolumn{2}{|c|}{ HRBC $(n=80)$} & \multirow{2}{*}{$\begin{array}{l}p \\
\text { value }\end{array}$} \\
\hline & $\begin{array}{l}\text { Positive } \\
(n=22)\end{array}$ & $\begin{array}{l}\text { Negative } \\
(n=39)\end{array}$ & & $\begin{array}{l}\text { Positive } \\
(n=15)\end{array}$ & $\begin{array}{l}\text { Negative } \\
(n=21)\end{array}$ & & $\begin{array}{l}\text { Positive } \\
(n=10)\end{array}$ & $\begin{array}{l}\text { Negative } \\
(n=70)\end{array}$ & \\
\hline \multirow{3}{*}{$\begin{array}{l}\text { Age at operation } \\
\leq 56 \\
>56\end{array}$} & \multirow{3}{*}{$\begin{array}{l}8(36.4 \%) \\
14(63.6 \%)\end{array}$} & \multirow{2}{*}{$\begin{array}{l}20 \\
(51.3 \%)\end{array}$} & \multirow[t]{3}{*}{0.262} & \multirow{3}{*}{$\begin{array}{l}5(33.3 \%) \\
10(66.7 \%)\end{array}$} & \multirow{2}{*}{$\begin{array}{l}11 \\
(52.4 \%)\end{array}$} & \multirow[t]{3}{*}{0.257} & \multirow{2}{*}{$\begin{array}{l}5 \\
(50.0 \%)\end{array}$} & \multirow{2}{*}{$\begin{array}{l}38 \\
(54.3 \%)\end{array}$} & \multirow[t]{3}{*}{0.799} \\
\hline & & & & & & & & & \\
\hline & & (48.7\%) & & & $\begin{array}{l}10 \\
(47.6 \%)\end{array}$ & & $\begin{array}{l}5 \\
(50.0 \%)\end{array}$ & $\begin{array}{l}32 \\
(45.7 \%)\end{array}$ & \\
\hline \multirow{3}{*}{$\begin{array}{l}\text { Menopause } \\
\text { Negative } \\
\text { Positive }\end{array}$} & \multirow{3}{*}{$\begin{array}{l}6(27.3 \%) \\
16(72.7 \%)\end{array}$} & \multirow{3}{*}{$\begin{array}{l}16 \\
(41.0 \%) \\
23 \\
(59.0 \%)\end{array}$} & \multirow[t]{3}{*}{0.283} & \multirow{3}{*}{$\begin{array}{l}4(26.7 \%) \\
11(73.3 \%)\end{array}$} & \multirow{3}{*}{$\begin{array}{l}10 \\
(47.6 \%) \\
11 \\
(52.4 \%)\end{array}$} & \multirow[t]{3}{*}{0.178} & \multirow{3}{*}{$\begin{array}{l}5 \\
(50.0 \%) \\
5 \\
(50.0 \%)\end{array}$} & \multirow{3}{*}{$\begin{array}{l}31 \\
(44.3 \%) \\
39 \\
(55.7 \%)\end{array}$} & \multirow[t]{3}{*}{0.734} \\
\hline & & & & & & & & & \\
\hline & & & & & & & & & \\
\hline Tumor size & $2(9.1 \%)$ & $5(12.8 \%)$ & 0.505 & $0(0.0 \%)$ & $6(28.6 \%)$ & \multirow[t]{2}{*}{0.028} & \multirow{2}{*}{$\begin{array}{l}3 \\
(30.0 \%) \\
7 \\
(70.0 \%)\end{array}$} & $8(11.4 \%)$ & \multirow[t]{2}{*}{0.136} \\
\hline $\begin{array}{l}\leq 2 \mathrm{~cm} \\
>2 \mathrm{~cm}\end{array}$ & $20(90.9 \%)$ & $\begin{array}{l}34 \\
(87.2 \%)\end{array}$ & & $\begin{array}{l}15 \\
(100.0 \%)\end{array}$ & $\begin{array}{l}15 \\
(71.4 \%)\end{array}$ & & & $\begin{array}{l}62 \\
(88.6 \%)\end{array}$ & \\
\hline Lymph node status & $2(9.1 \%)$ & $9(23.1 \%)$ & 0.155 & $6(40.0 \%)$ & $5(23.8 \%)$ & 0.298 & 4 & 15 & 0.183 \\
\hline $\begin{array}{l}\text { Negative } \\
\text { Positive }\end{array}$ & $20(90.9 \%)$ & $\begin{array}{l}30 \\
(76.9 \%)\end{array}$ & & $9(60.0 \%)$ & $\begin{array}{l}16 \\
(76.2 \%)\end{array}$ & & $\begin{array}{l}6 \\
(60.0 \%)\end{array}$ & $\begin{array}{l}55 \\
(78.6 \%)\end{array}$ & \\
\hline Nuclear grade & $16(72.7 \%)$ & 28 & 0.938 & $13(86.7 \%)$ & $\begin{array}{l}15 \\
(714 \%)\end{array}$ & 0.253 & 9 & 56 & 0.400 \\
\hline 1,2 & $6(27.3 \%)$ & & & $2(13.3 \%)$ & & & & & \\
\hline 3 & & $(28.2 \%)$ & & & $\mathrm{b}(28.0 \%)$ & & $(10.0 \%)$ & $\begin{array}{l}14 \\
(20.0 \%)\end{array}$ & \\
\hline Ki67 & $6(27.3 \%)$ & $\begin{array}{l}12 \\
(30.8 \%)\end{array}$ & 0.774 & $9(60.0 \%)$ & $8(38.1 \%)$ & 0.194 & $\begin{array}{l}7 \\
(70.0 \%)\end{array}$ & $\begin{array}{l}32 \\
(45.7 \%)\end{array}$ & 0.136 \\
\hline$\leq 14 \%$ & $16(72.7 \%)$ & & & $6(40.0 \%)$ & 13 & & & & \\
\hline$>14 \%$ & & $(69.2 \%)$ & & & & & $(30.0 \%)$ & $(54.3 \%)$ & \\
\hline $\begin{array}{l}\text { Pathological } \\
\text { resnonse }\end{array}$ & $3(13.6 \%)$ & 25 & $<$ & $4(26.7 \%)$ & 14 & 0.020 & 1 & 20 & 0.198 \\
\hline $\begin{array}{l}\text { pCR } \\
\text { non-pCR }\end{array}$ & 19 (86.4\%) & $\begin{array}{l}14 \\
(35.9 \%)\end{array}$ & & 11 (73.3\%) & 7 (33.3\%) & & $\begin{array}{l}9 \\
(90.0 \%)\end{array}$ & $\begin{array}{l}50 \\
(71.4 \%)\end{array}$ & \\
\hline
\end{tabular}

\section{LAG-3 expression in HER2 enriched breast cancer}

Among the 36 HER2BC patients, 15 (41.7\%) were in the high-LAG-3 expression group, and 21 (58.3\%) were in the low-LAG-3 expression group. In the clinicopathological examination, tumor diameter was significantly smaller in the LAG-3 low-expression group than in the high-expression group $(p=0.028)$, and the number of $p C R$ patients was greater $(p=0.020)$ (Table 3$)$. Outcome analysis showed significantly longer DFS ( $p=0.013$, log-rank) (Fig. $2 C$ ) and OS ( $p=0.003$, log-rank) (Fig. $3 C$ ) in the low-LAG-3 expression group than that in the high-LAG-3 expression group. On univariate analysis for recurrence, low-LAG-3 expression status was a good prognostic factor $(p=0.042, H R=10.969)$. Multivariate analysis also showed that low-LAG-3 expression status was an independent good prognostic factor $(p=0.048, \mathrm{HR}=10.400)$ (Table 2$)$.

\section{LAG-3 expression in hormone receptor-positive breast cancer}

Among the 80 HRBC patients, 10 (12.5\%) were in the high-LAG-3 expression group, and 70 (87.5\%) were in the low-LAG-3 expression group. No correlation was found between LAG-3 expression and any clinicopathological factor in HRBC patients (Table 3) or between 
LAG-3 expression and outcomes (Table 2) (Fig. 2D) (Fig. 3D).

\section{Discussion}

In the past it was believed that cancer cells could autonomously proliferate and survive because of a variety of genetic abnormalities, but recently it has become clear that the peripheral environment (tumor microenvironment) greatly affects cancer cells and contributes to the formation of characteristics particular to cancer [40]. Improvement of the immune microenvironment in tumor is needed because the immune microenvironment in tumor tissues affects not only the efficacy of immunotherapy, but also the efficacy and prognosis of chemotherapy and other modes of anticancer therapy [22, 23]. Tumor antigens are present on tumor cells, and they induce an antitumor immune response in the host. Moreover, tumors activate mechanisms to suppress antitumor immunity, particularly in the microenvironment of tumor tissue, and they utilize those mechanisms for self-proliferation [1]. Furthermore, there are diverse activation and suppression signals for antitumor immunity, and they are integrated to adjust the T-cell activation process [3]. In the group of immunoadjuvant molecules that regulate T-cell activation, inhibitory molecules such as CTLA-4, PD-1, PD-L1/2, LAG-3, and TIM-3 function as immunologic checkpoints $[14,18]$. Furthermore, immunotherapy utilizing a blocking antibody to inhibit the signals of the immunological checkpoints has shown promising therapeutic efficacy in a clinical setting $[5,7,8]$. Meanwhile, activators include 0X-40, and it has been reported that administration of an anti-0X-40 agonistic antibody may enhance the antitumor immune response [41]. In cancers such as malignant melanoma, renal cell carcinoma, and breast cancer, a correlation has been suggested between PD-1 and PD-L1 expression levels and both the malignancy of cancer and extent of a poor prognosis [9, 11, 22]. However, there have been few reports that have examined the level of expression of immunoadjuvant molecules such as LAG-3, TIM-3, and OX40 clinically, and therefore monitoring of the tumor immune microenvironment has been conducted in NAC breast cancer patients using TILs. In this study, the LAG low-expression group had a significantly higher pCR rate than the high LAG expression group in NAC breast cancer patients, and low-LAG expression contributed to an extension of the disease-free survival interval.

LAG-3 is a molecule with a structure similar to CD4, and it appears on the cell surface when T-cells are activated $[13,15,42]$. The signal transduction pathway of LAG-3 is still unclear, but it is believed to function as a molecule that not only suppresses the proliferation and activation of T-cells, but that also plays a key role as an immune checkpoint similar to PD-1 and CTLA-4 [14]. Basic study has demonstrated that the antitumor immune response in mice is enhanced by inhibiting the LAG-3 signal using an anti-LAG-3 antibody while concurrently administering an anti-PD-1 antibody [43]. Furthermore, an anti-LAG-3 antibody was adapted for human use, and it was discovered that the use of this antibody in combination with paclitaxel in phase I and II clinical studies of breast cancer raised the response rate from $25-50 \%$ compared with groups treated with anticancer monotherapy [44]. Paclitaxel is considered to improve immune escape in the host by suppressing Tregs, but expression of LAG-3 has also been found in Tregs. In other words, an anti-LAG-3 antibody in combination with paclitaxel may effectively act to relieve immunosuppression by suppressing Tregs. In the NAC regimen used in our study that treats paclitaxel as a key drug, it appears that these mechanisms enable immune response monitoring via LAG3 expression.

On the other hand, TIM-3 has galectin-9 as a ligand and suppresses the activation of effector T-cells mediated by ligand-receptor interaction [16-18]. Clinical studies have reported that the level of expression of TIM-3 in renal cell carcinoma and head and neck cancer exhibits a negative correlation to prognosis $[45,46]$. In our study, we found no correlation between TIM-3 expression and the therapeutic efficacy of NAC. TIM-3 is a molecule that contributes to the suppression of T-cell function synergistically with PD-1, and simply activating antitumor immunity by lowering TIM-3 expression alone may not improve the tumor immune microenvironment. Moreover, OX-40 is a member of the TNF receptor superfamily, and is expressed by activated T-cells, NK cells, and Tregs [19-21]. It has been reported in studies using a mouse model that administration of an anti-OX-40 agonistic antibody enabled rejection of fully established tumors [41]. In our study, however, we found no correlation between OX-40 expression and therapeutic efficacy of NAC. Under the assumption that no potent antitumor effect will be obtained with anti-0X-40 agonistic antibody monotherapy, a search is now underway in basic study for a combination therapy with another drug [47]. In other words, in the case of OX-40 as well, this finding indicates that a clear improvement of the tumor immune microenvironment cannot be obtained clinically through modulation of OX-40 expression alone. In addition, no correlations between expression of LAG-3, TIM-3, and OX-40 were found in this study.

In previous study, breast cancer was not considered as a cancer that develops as the result of an immune disorder [48]. Recently, however, breast cancer has come to be viewed as an immunogenic tumor, and the highly malignant subtypes TNBC and HER2BC have a high level of immune activity $[49,50]$. We believe that in these highly malignant breast cancers the tumor immune microenvironment can be reliably monitored by TILs because of the high immune activity. In our study, when breast cancer was stratified by intrinsic

Page $10 / 17$ 
subtype and TILs were evaluated, the groups with low-LAG expression in highly malignant breast cancers had a significantly higher PCR rate, but no significant difference was found for HRBC.

\section{Conclusion}

Our findings suggest that LAG-3 may become a biomarker in highly malignant breast cancers such as TNBC and HER2BC that can predict the therapeutic efficacy of NAC.

\section{List Of Abbreviations}

CTLA- 4

cytotoxic T-lymphocyte-associated antigen 4; PD-1:programmed cell death 1; BC:breast cancer; TNBC:triple-negative breast cancer; PDL:programmed cell death-ligand; NAC:neoadjuvant chemotherapy; LAG-3:Lymphocyte activation gene-3; TIM-3:T-cell immunoglobulin and mucin containing protein-3; OX-40:orexin-40;TILs:tumor-infiltrating lymphocytes; Tregs:regulatory T cells; CT:computed tomography; US:ultrasonography; ER:estrogen receptor; PgR:progesteron receptor; HER2:human epidermal growth factor receptor 2; HER2BC:HER2-enriched breast cancer; HRBC:hormone receptor-positive breast cancer; RECIST:Response Evaluation Criteria in Solid Tumors; pCR:pathological complete response; PD:progressive disease; OS:Overall survival; DFS:Disease-free survival; FISH:fluorescent in situ hybridization; CEP17:centromere 17; HE:hematoxylin and eosin; FOVs:fields of view; HR:hazards ratios; c.i.:confidence interval; PR:partial response; SD:stable disease; TNF:tissue necrosis factor; NK:natural killer

\section{Declarations}

\section{Ethics approval and consent to participate}

A written informed consent to participate in the study was obtained from each subject in accordance with the declaration of Helsinki principles. Each patient or the patient's family was fully informed of the investigational nature of this study and provided their written, informed consent. The study protocol was approved by the Ethics Committee of Osaka City University (approve number \#926).

\section{Consent for publication}

Not applicable.

Availability of data and materials

The datasets used and/or analyzed during the current study are available from the corresponding author on reasonable request.

\section{Competing interests}

The authors declare that they have no competing interests.

\section{Funding}

This study was supported in part by Grants-in Aid for Scientific Research (KAKENHI, Nos. 19K18067, 17K10559, and 20K08938) from the Ministry of Education, Science, Sports, Culture and Technology of Japan.

\section{Authors' contributions}

All authors were involved in the preparation of this manuscript. YA collected the data and wrote the manuscript. SK, SI, KT, WG, and TM performed the operation and designed the study. YA and SK summarized the data and revised the manuscript. MS, HT, KH, and MO provided a substantial contribution to the study design, performed the operation, and revised the manuscript. All authors read and approved the final manuscript.

\section{Acknowledgements}

We thank Yayoi Matsukiyo and Tomomi Okawa (Department of Breast and Endocrine Surgery, Osaka City University Graduate School of Medicine) for helpful advice regarding data management. 
Author's information

${ }^{1}$ Department of Breast and Endocrine Surgery, Osaka City University Graduate School of Medicine, 1-4-3 Asahi-machi, Abeno-ku, Osaka 545-8585, Japan.

${ }^{2}$ Department of Gastrointestinal Surgery, Osaka City University Graduate School of Medicine, 1-4-3 Asahi-machi, Abeno-ku, Osaka 5458585, Japan.

\section{References}

1. Schreiber RD, Old LJ, Smyth MJ: Cancer immunoediting: integrating immunity's roles in cancer suppression and promotion. Science 2011, 331(6024):1565-1570.

2. Couzin-Frankel J: Breakthrough of the year 2013. Cancer immunotherapy. Science 2013, 342(6165):1432-1433.

3. Chen L, Flies DB: Molecular mechanisms of T cell co-stimulation and co-inhibition. Nat Rev Immuno/ 2013, 13(4):227-242.

4. Iwai Y, Ishida M, Tanaka Y, Okazaki T, Honjo T, Minato N: Involvement of PD-L1 on tumor cells in the escape from host immune system and tumor immunotherapy by PD-L1 blockade. Proc Natl Acad Sci U S A 2002, 99(19):12293-12297.

5. Robert C, Long GV, Brady B, Dutriaux C, Maio M, Mortier L, Hassel JC, Rutkowski P, McNeil C, Kalinka-Warzocha E et al: Nivolumab in previously untreated melanoma without BRAF mutation. N Engl J Med 2015, 372(4):320-330.

6. Wolchok JD, Kluger H, Callahan MK, Postow MA, Rizvi NA, Lesokhin AM, Segal NH, Ariyan CE, Gordon RA, Reed K et al: Nivolumab plus ipilimumab in advanced melanoma. N Engl J Med 2013, 369(2):122-133.

7. Topalian SL, Sznol M, McDermott DF, Kluger HM, Carvajal RD, Sharfman WH, Brahmer JR, Lawrence DP, Atkins MB, Powderly JD et al: Survival, durable tumor remission, and long-term safety in patients with advanced melanoma receiving nivolumab. $J$ Clin Oncol 2014, 32(10):1020-1030.

8. Momtaz P, Postow MA: Immunologic checkpoints in cancer therapy: focus on the programmed death-1 (PD-1) receptor pathway. Pharmgenomics Pers Med 2014, 7:357-365.

9. Muenst S, Soysal SD, Gao F, Obermann EC, Oertli D, Gillanders WE: The presence of programmed death 1 (PD-1)-positive tumorinfiltrating lymphocytes is associated with poor prognosis in human breast cancer. Breast Cancer Res Treat 2013, 139(3):667676.

10. Sun S, Fei X, Mao Y, Wang X, Garfield DH, Huang O, Wang J, Yuan F, Sun L, Yu Q et al: PD-1(+) immune cell infiltration inversely correlates with survival of operable breast cancer patients. Cancer Immunol Immunother 2014, 63(4):395-406.

11. Muenst S, Schaerli AR, Gao F, Daster S, Trella E, Droeser RA, Muraro MG, Zajac P, Zanetti R, Gillanders WE et al: Expression of programmed death ligand 1 (PD-L1) is associated with poor prognosis in human breast cancer. Breast Cancer Res Treat 2014, 146(1):15-24.

12. Stagg J, Allard B: Immunotherapeutic approaches in triplenegative breast cancer: latest research and clinical prospects. Ther $A d v$ Med Oncol 2013, 5(3):169-181.

13. Triebel F, Jitsukawa S, Baixeras E, Roman-Roman S, Genevee C, Viegas-Pequignot E, Hercend T: LAG-3, a novel lymphocyte activation gene closely related to CD4. J Exp Med 1990, 171(5):1393-1405.

14. Nguyen LT, Ohashi PS: Clinical blockade of PD1 and LAG3-potential mechanisms of action. Nat Rev Immuno/2015, 15(1):4556.

15. Demeure CE, Wolfers J, Martin-Garcia N, Gaulard P, Triebel F: T Lymphocytes infiltrating various tumour types express the MHC class II ligand lymphocyte activation gene-3 (LAG-3): role of LAG-3/MHC class II interactions in cell-cell contacts. Eur J Cancer 2001, 37(13):1709-1718.

16. Monney L, Sabatos CA, Gaglia JL, Ryu A, Waldner H, Chernova T, Manning S, Greenfield EA, Coyle AJ, Sobel RA et al: Th1-specific cell surface protein Tim-3 regulates macrophage activation and severity of an autoimmune disease. Nature 2002, 415(6871):536-541.

17. Anderson AC, Anderson DE, Bregoli L, Hastings WD, Kassam N, Lei C, Chandwaskar R, Karman J, Su EW, Hirashima M et al: Promotion of tissue inflammation by the immune receptor Tim-3 expressed on innate immune cells. Science 2007, 318(5853):1141-1143.

18. Ngiow SF, Teng MW, Smyth MJ: Prospects for TIM3-Targeted Antitumor Immunotherapy. Cancer Res 2011, 71(21):6567-6571. 
19. Paterson DJ, Jefferies WA, Green JR, Brandon MR, Corthesy P, Puklavec M, Williams AF: Antigens of activated rat T lymphocytes including a molecule of 50,000 Mr detected only on CD4 positive T blasts. Mol Immunol 1987, 24(12):1281-1290.

20. Vetto JT, Lum S, Morris A, Sicotte M, Davis J, Lemon M, Weinberg A: Presence of the T-cell activation marker OX-40 on tumor infiltrating lymphocytes and draining lymph node cells from patients with melanoma and head and neck cancers. Am J Surg 1997, 174(3):258-265.

21. Curti BD, Kovacsovics-Bankowski M, Morris N, Walker E, Chisholm L, Floyd K, Walker J, Gonzalez I, Meeuwsen T, Fox BA et al: OX40 is a potent immune-stimulating target in late-stage cancer patients. Cancer Res 2013, 73(24):7189-7198.

22. Fridman WH, Pages F, Sautes-Fridman C, Galon J: The immune contexture in human tumours: impact on clinical outcome. Nat Rev Cancer 2012, 12(4):298-306.

23. Zitvogel L, Kepp O, Kroemer G: Immune parameters affecting the efficacy of chemotherapeutic regimens. Nat Rev Clin Oncol 2011, 8(3):151-160.

24. Liu H, Zhang T, Ye J, Li H, Huang J, Li X, Wu B, Huang X, Hou J: Tumor-infiltrating lymphocytes predict response to chemotherapy in patients with advance non-small cell lung cancer. Cancer Immunol Immunother 2012, 61(10):1849-1856.

25. Kocian P, Sedivcova M, Drgac J, Cerna K, Hoch J, Kodet R, Bartunkova J, Spisek R, Fialova A: Tumor-infiltrating lymphocytes and dendritic cells in human colorectal cancer: their relationship to KRAS mutational status and disease recurrence. Hum Immunol 2011, 72(11):1022-1028.

26. Lee WS, Kang M, Baek JH, Lee JI, Ha SY: Clinical impact of tumor-infiltrating lymphocytes for survival in curatively resected stage IV colon cancer with isolated liver or lung metastasis. Ann Surg Oncol 2013, 20(2):697-702.

27. Ainbinder DJ, Esmaeli B, Groo SC, Finger PT, Brooks JP: Introduction of the 7th edition eyelid carcinoma classification system from the American Joint Committee on Cancer-International Union Against Cancer staging manual. Arch Pathol Lab Med 2009, 133(8):1256-1261.

28. Mauri D, Pavlidis N, loannidis JP: Neoadjuvant versus adjuvant systemic treatment in breast cancer: a meta-analysis. $J$ Nat/ Cancer Inst 2005, 97(3):188-194.

29. Mieog JS, van der Hage JA, van de Velde CJ: Preoperative chemotherapy for women with operable breast cancer. Cochrane Database Syst Rev 2007(2):CD005002.

30. Buzdar AU, Valero V, Ibrahim NK, Francis D, Broglio KR, Theriault RL, Pusztai L, Green MC, Singletary SE, Hunt KK et al: Neoadjuvant therapy with paclitaxel followed by 5-fluorouracil, epirubicin, and cyclophosphamide chemotherapy and concurrent trastuzumab in human epidermal growth factor receptor 2-positive operable breast cancer: an update of the initial randomized study population and data of additional patients treated with the same regimen. Clin Cancer Res 2007, 13(1):228-233.

31. Eisenhauer EA, Therasse P, Bogaerts J, Schwartz LH, Sargent D, Ford R, Dancey J, Arbuck S, Gwyther S, Mooney M et al: New response evaluation criteria in solid tumours: revised RECIST guideline (version 1.1). Eur J Cancer 2009, 45(2):228-247.

32. Kashiwagi S, Yashiro M, Takashima T, Aomatsu N, Kawajiri H, Ogawa Y, Onoda N, Ishikawa T, Wakasa K, Hirakawa K: c-Kit expression as a prognostic molecular marker in patients with basal-like breast cancer. Br J Surg 2013, 100(4):490-496.

33. Umemura S, Kurosumi M, Moriya T, Oyama T, Arihiro K, Yamashita H, Umekita Y, Komoike Y, Shimizu C, Fukushima H et al: Immunohistochemical evaluation for hormone receptors in breast cancer: a practically useful evaluation system and handling protocol. Breast Cancer 2006, 13(3):232-235.

34. Wolff AC, Hammond ME, Hicks DG, Dowsett M, McShane LM, Allison KH, Allred DC, Bartlett JM, Bilous M, Fitzgibbons P et al: Recommendations for human epidermal growth factor receptor 2 testing in breast cancer: American Society of Clinical Oncology/College of American Pathologists clinical practice guideline update. J Clin Oncol 2013, 31(31):3997-4013.

35. Wolff AC, Hammond ME, Hicks DG, Dowsett M, McShane LM, Allison KH, Allred DC, Bartlett JM, Bilous M, Fitzgibbons P et al: Recommendations for human epidermal growth factor receptor 2 testing in breast cancer: American Society of Clinical Oncology/College of American Pathologists clinical practice guideline update. Arch Pathol Lab Med 2014, 138(2):241-256.

36. Goldhirsch A, Wood WC, Coates AS, Gelber RD, Thurlimann B, Senn HJ: Strategies for subtypes-dealing with the diversity of breast cancer: highlights of the St. Gallen International Expert Consensus on the Primary Therapy of Early Breast Cancer 2011. Ann Oncol 2011, 22(8):1736-1747.

37. Salgado R, Denkert C, Demaria S, Sirtaine N, Klauschen F, Pruneri G, Wienert S, Van den Eynden G, Baehner FL, Penault-Llorca F et al: The evaluation of tumor-infiltrating lymphocytes (TILs) in breast cancer: recommendations by an International TILs Working Group 2014. Ann Oncol 2015, 26(2):259-271. 
38. Ono M, Tsuda H, Shimizu C, Yamamoto S, Shibata T, Yamamoto H, Hirata T, Yonemori K, Ando M, Tamura K et al: Tumorinfiltrating lymphocytes are correlated with response to neoadjuvant chemotherapy in triple-negative breast cancer. Breast Cancer Res Treat 2012, 132(3):793-805.

39. Mao Y, Qu Q, Zhang Y, Liu J, Chen X, Shen K: The value of tumor infiltrating lymphocytes (TILs) for predicting response to neoadjuvant chemotherapy in breast cancer. a systematic review and meta-analysis. PLoS One 2014, 9(12):e115103.

40. Hanahan D, Weinberg RA: Hallmarks of cancer: the next generation. Cell 2011, 144(5):646-674.

41. Weinberg AD, Rivera MM, Prell R, Morris A, Ramstad T, Vetto JT, Urba WJ, Alvord G, Bunce C, Shields J: Engagement of the OX-40 receptor in vivo enhances antitumor immunity. $J$ Immunol 2000, 164(4):2160-2169.

42. Baixeras E, Huard B, Miossec C, Jitsukawa S, Martin M, Hercend T, Auffray C, Triebel F, Piatier-Tonneau D: Characterization of the lymphocyte activation gene 3 -encoded protein. A new ligand for human leukocyte antigen class II antigens. J Exp Med 1992, 176(2):327-337.

43. Okazaki T, Okazaki IM, Wang J, Sugiura D, Nakaki F, Yoshida T, Kato Y, Fagarasan S, Muramatsu M, Eto T et al: PD-1 and LAG-3 inhibitory co-receptors act synergistically to prevent autoimmunity in mice. J Exp Med 2011, 208(2):395-407.

44. Brignone C, Gutierrez M, Mefti F, Brain E, Jarcau R, Cvitkovic F, Bousetta N, Medioni J, Gligorov J, Grygar C et al: First-line chemoimmunotherapy in metastatic breast carcinoma: combination of paclitaxel and IMP321 (LAG-3lg) enhances immune responses and antitumor activity. J Trans/ Med 2010, 8:71.

45. Yuan J, Jiang B, Zhao H, Huang Q: Prognostic implication of TIM-3 in clear cell renal cell carcinoma. Neoplasma 2014, 61(1):3540.

46. Cao Y, Zhou X, Huang X, Li Q, Gao L, Jiang L, Huang M, Zhou J: Tim-3 expression in cervical cancer promotes tumor metastasis. PLoS One 2013, 8(1):e53834.

47. Hirschhorn-Cymerman D, Budhu S, Kitano S, Liu C, Zhao F, Zhong H, Lesokhin AM, Avogadri-Connors F, Yuan J, Li Y et al: Induction of tumoricidal function in CD4 + T cells is associated with concomitant memory and terminally differentiated phenotype. J Exp Med 2012, 209(11):2113-2126.

48. Rosenberg SA, Yang JC, Restifo NP: Cancer immunotherapy: moving beyond current vaccines. Nat Med 2004, 10(9):909-915.

49. Adams S, Gray RJ, Demaria S, Goldstein L, Perez EA, Shulman LN, Martino S, Wang M, Jones VE, Saphner TJ et al: Prognostic value of tumor-infiltrating lymphocytes in triple-negative breast cancers from two phase III randomized adjuvant breast cancer trials: ECOG 2197 and ECOG 1199. J Clin Oncol 2014, 32(27):2959-2966.

50. Denkert C, von Minckwitz G, Brase JC, Sinn BV, Gade S, Kronenwett R, Pfitzner BM, Salat C, Loi S, Schmitt WD et al: Tumorinfiltrating lymphocytes and response to neoadjuvant chemotherapy with or without Carboplatin in human epidermal growth factor receptor 2-positive and triple-negative primary breast cancers. J Clin Oncol 2015, 33(9):983-991.

\section{Supplemental Figure}

Supplemental Figure is not available with this version

\section{Figures}




\section{Fig. 1 Asano Y. et al.}

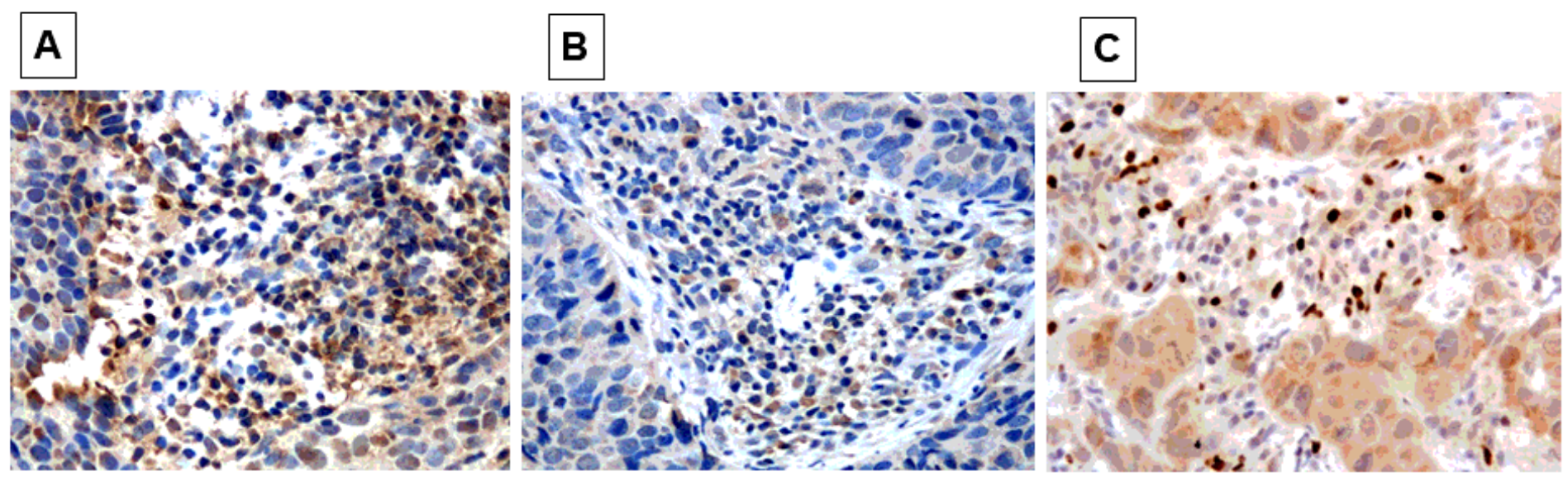

\section{Figure 1}

Immunohistochemical determination. To evaluate LAG-3 (A), TIM-3 (B), and OX-40 (C) expression, the number of infiltrating Tlymphocytes stained with anti-LAG-3, anti-TIM-3, and anti-OX-40 antibody in areas surrounding cancer cells was measured under 400times magnified microscopy in each of 5 fields of view (FOVs) selected in darkly stained areas. The median value of the average number in the 5 FOVs was determined, and that number was set as a cut-off value. 
Fig. 2 Asano Y. et al.
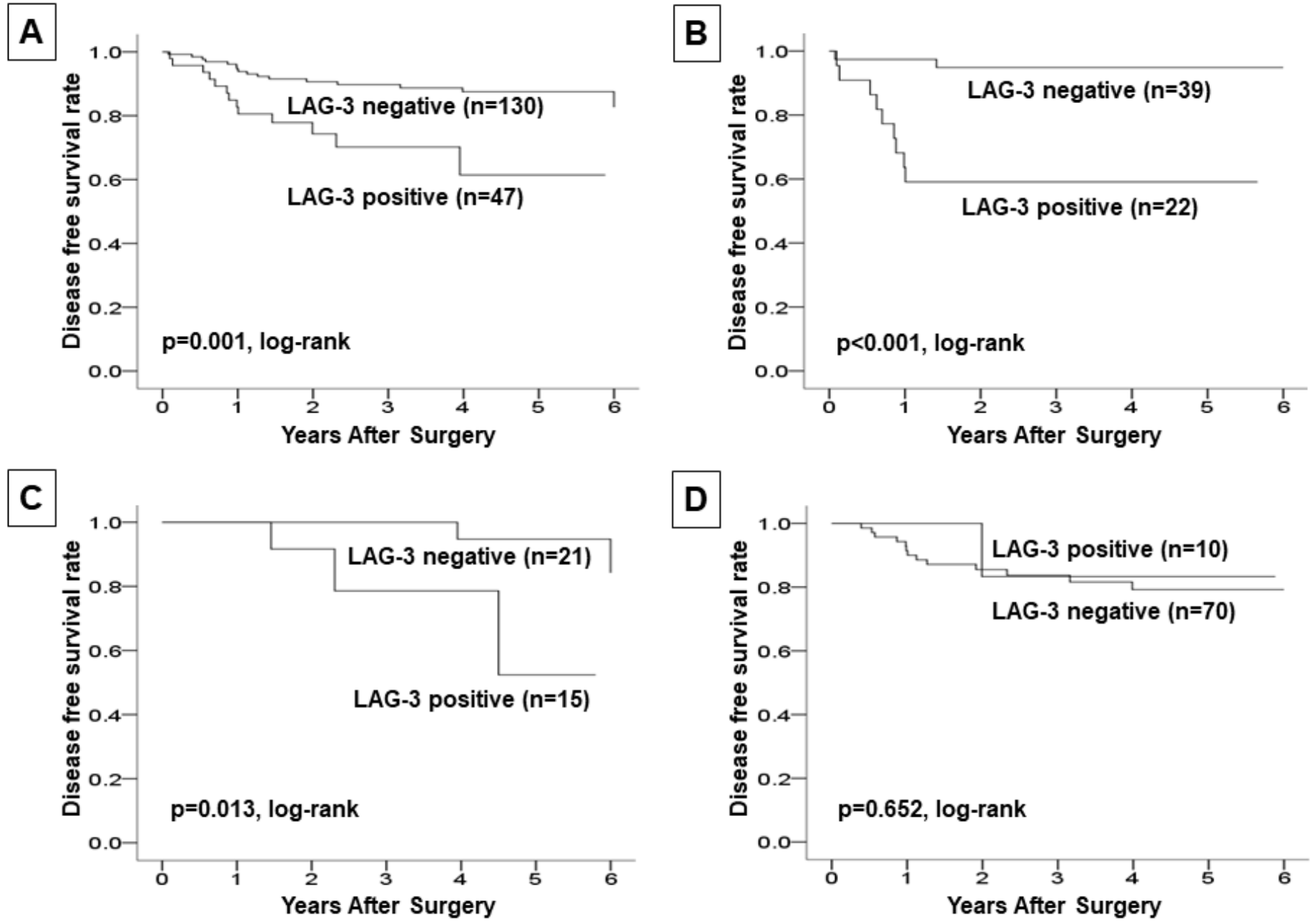

Figure 2

Disease-free survival of the patients with breast cancer by subtypes based on the LAG-3 expression. We found a significant DFS extension in the LAG-3 low-expression group over the high-expression group in 177 all breast cancer patients $(p=0.001$, log-rank) $(A)$. In TNBC and HER2BC, outcome analysis showed significantly longer DFS in the low-LAG-3 expression group than that in the high-LAG-3 expression group ( $<0.001$, log-rank) (B) ( $p=0.013$, log-rank) (C). DFS did not differ significantly between patients with low vs high LAG-3 expression ( $p=0.652$, log-rank) (D). 
Fig. 3 Asano Y. et al.
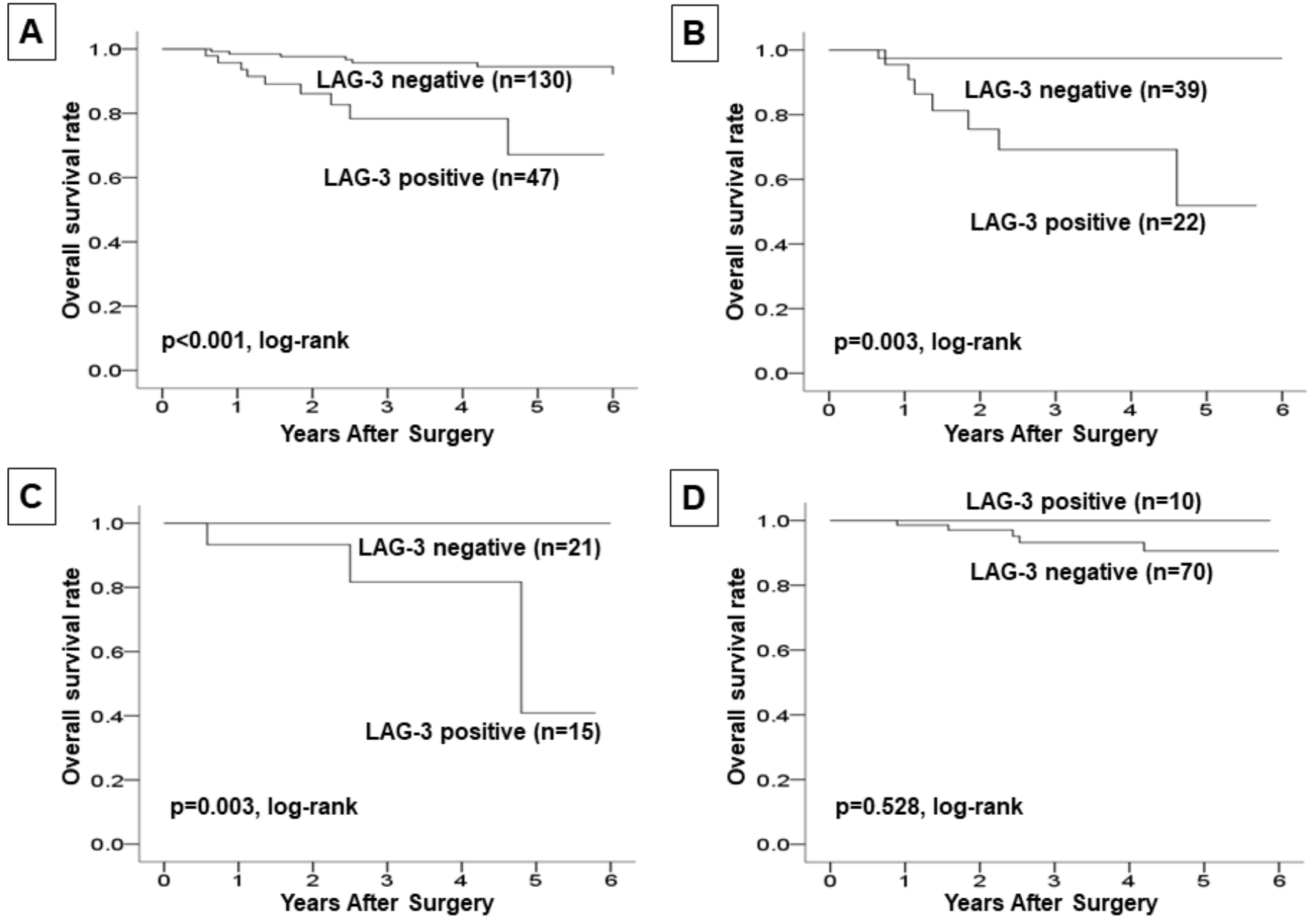

Figure 3

Overall survival of the patients with breast cancer by subtypes based on the LAG-3 expression. We found a significant OS extension in the LAG-3 low-expression group over the high-expression group in 177 all breast cancer patients $(p<0.001$, log-rank) ( $A$ ). In TNBC and HER2BC, outcome analysis showed significantly longer OS in the low-LAG-3 expression group than that in the high-LAG-3 expression group ( $p=0.003$, log-rank) (B) ( $p=0.003$, log-rank) (C). OS did not differ significantly between patients with low vs high LAG-3 expression ( $p=0.528$, log-rank) $(D)$.

\section{Supplementary Files}

This is a list of supplementary files associated with this preprint. Click to download.

- STROBEchecklistcohort.docx 